Check for updates

Cite this: RSC Adv., 2017, 7, 51750

Received 2nd July 2017

Accepted 27th September 2017

DOI: $10.1039 / \mathrm{c} 7 \mathrm{ra0} 3304 f$

rsc.li/rsc-advances

\section{Transmembrane oligomeric intermediates of pore forming toxin Cytolysin A determine leakage kinetics $\uparrow$}

\begin{abstract}
Ayush Agrawal, ${ }^{a}$ K. Apoorva ${ }^{b}$ and K. G. Ayappa (iD *ac
Calcein leakage experiments for the $\alpha$ pore forming toxin Cytolysin A (ClyA) are carried out using a suspension of small unilamellar vesicles made up of 1,2-dipalmitoyl-sn-glycero-3-phosphocholine (DPPC) and cholesterol. Combining the oligomerization kinetics with a Poisson process to describe the inherent stochasticity underlying pore formation, we screen possible oligomerization pathways by comparing model predictions with calcein leakage data for ClyA. Excellent agreement with the leakage data was obtained for a reversible sequential oligomerization mechanism upon inclusion of leakage from membrane inserted partially oligomerized intermediates or 'arcs'. In contrast, the non-sequential mechanisms were unable to predict the calcein leakage data. Reversibility in the oligomerization mechanism maintains a constant supply of protomers resulting in a broad distribution of oligomers at steady state. Additionally, the time scale for the conformational change from the water soluble monomer to the membrane bound protomer was found to be similar or larger than the time scale for oligomerization. The dominant contribution to leakage was found to occur from the smaller arcs, consistent with the low protein to lipid ratios used in the experiment. Our kinetic model is able to capture both the fast and slow time constants typically observed in calcein leakage experiments. A key inference is that arcs play a critical role in the leakage kinetics of ClyA, with the fast leakage time scale arising from the smaller oligomerized intermediates and the longer time scale arising from the slowly forming higher order oligomers.
\end{abstract}

\section{Introduction}

Pore forming toxins (PFTs) are specific proteins expressed by a wide variety of organisms which attack target cells by rapid and unregulated pore formation. In addition to determining the crystal structure of the pore complex and conformational changes that accompany binding and pore formation, there is substantial interest in unraveling the kinetics and oligomerization pathways for these toxins. A knowledge of pore formation kinetics and oligomerization pathways could potentially help in developing intervention strategies to prevent pore formation, mitigate PFT mediated infections as well as use PFTs for technological applications such as DNA sequencing. ${ }^{1}$

The pre-pore and growing pore models are the two main pore forming paradigms that have been proposed for pore assembly

${ }^{a}$ Department of Chemical Engineering, Indian Institute of Science, Bangalore-560012, India.E-mail:ayappa@iisc.ac.in

${ }^{b}$ Department of Chemical Engineering, Indian Institute of Technology, Hyderabad502205, India

${ }^{c}$ Centre for Biosystems Science and Engineering, Indian Institute of Science, Bangalore560012, India

$\dagger$ Electronic supplementary information (ESI) available. See DOI: $10.1039 / \mathrm{c} 7 \mathrm{ra} 07304 \mathrm{f}$ pathways. ${ }^{2}$ In the pre-pore model a fully assembled pore or oligomer assembles on the membrane interface and converts to form a functional membrane inserted pore complex. Several $\beta$ toxins such as listeriolysin O (LLO), lysenin, $\alpha$-hemolysin and perfringolysin ${ }^{3}$ are known to form a pre-pore complex, verified by monitoring changes in height along the bilayer normal using atomic force microscopy (AFM). ${ }^{4}$ More recently high speed AFM with sub-second scanning time reveal the kinetics of these changes. ${ }^{5}$ In the growing pore model, membrane inserted intermediates known as 'arcs' devoid of lipid in the inner lumen undergo oligomerization to form the pore complex. These arcs can initiate lysis or leakage during the oligomerization process en route to forming the complete pore complex. Presence of arcs naturally raises the question of how arcs are formed. In one scenario arcs can form due to the oligomerization of membrane inserted protomers. Arcs if stable, would be capable of leakage due to their membrane inserted state. In an alternate scenario arcs can be formed by a 'pre-arc' state wherein the oligomeric intermediate first assembles on the membrane interface and subsequently undergoes an insertion to create a functional arc. Inserted arcs as well as pre-arcs at different stages of oligomerization have been observed in high AFM images of LLO., ${ }^{4,6-8}$ Since arcs are oligomerization intermediates their population on the membrane surface could in principle be modulated by the 
surface concentration of bound protein. It has been indicated that arcs are more likely to occur at low toxin concentration. ${ }^{9}$

Although detailed models for oligomerization kinetics of $\beta$ toxins are yet to appear in the literature, structural data gleaned from AFM images suggest a variety of intermediates that could play a role during pore formation. The situation is less certain when once considers $\alpha$-toxins which is the subject of this manuscript. In contrast to $\beta$-toxins which benefit from the increased stability imparted by interstrand hydrogen bonding in the membrane inserted $\beta$-barrel, $\alpha$-toxins form pores with an assembly of amphiphatic $\alpha$-helices. As a consequence there have been fewer crystal structures determined for the $\alpha$-toxins. The crystal structure of Cytolysin A (ClyA) expressed by E. coli, has been recently elucidated and a pore formation pathway based on the pre-pore model was hypothesized. ${ }^{10}$ Pore formation in the case of ClyA, is thought to occur through the following sequence of steps. Initially the water soluble monomer converts to a membrane bound protomer through a large conformational change during which the $\mathrm{N}$-terminus moves out of a dominant helical bundle present in the monomer. The initial binding to the membrane is thought to occur through the hydrophobic $\beta$-tongue and recent molecular dynamics simulations illustrate this initial step, ${ }^{\mathbf{1 1}}$ which is followed by the insertion of the N-terminus into the membrane. The details of subsequent oligomerization steps which lead to the formation of the dodecameric pore complex are unclear; oligomerization can either occur with the $\mathrm{N}$-terminus present at the membrane interface (pre-pore model) or can proceed with the N-terminus inserted into the membrane (growing pore model). Conductance experiments with the $\alpha$ PFT, equinatoxin II (EqtII) revealed much broader conductance distributions with greater irregularity in the conductance signals when compared with the $\beta$-toxin, $\gamma$-hemolysin. ${ }^{12}$ Pores with EqtII were purported to form structures where part of the pore channel consisted of the $\alpha$ toxins with toroidal lipids making up parts of the channel where proteins were absent. Using X-ray crystallography, lipidic pore arrangements have been observed in pore forming peptides. ${ }^{13}$ Pores formed with participating lipids could be more general and pertinent to pores formed by $\alpha$ toxins.

There have been several studies in the literature where pore formation rates have been investigated. ${ }^{\mathbf{1 4 , 1 5}} \mathrm{A}$ majority of the experimental work has been carried out on SUVs where dye leakage is on the order of a few $\mathrm{ms}$ and the rates of pore formation are controlling. ${ }^{16}$ Typical dye leakage from a suspension of SUVs have a characteristic feature: a fast time constant at early times followed by a longer and slower time constant. ${ }^{16,17}$ Pore formation rates are usually extracted from the fast time constant, ${ }^{\mathbf{1 8}}$ by assuming a Poisson process governing the arrival rates of pores on the vesicles. In contrast to experiments with SUVs, leakage experiments with single GUVs appear to have greater control as leakage from GUVs can be directly imaged. ${ }^{19,20}$ Since diffusion from GUVs is rate controlling, kinetics obtained from single GUV leakage experiments can be related to the number of pores formed on the vesicle at steady leakage rates provided a precise knowledge of the relation between toxin concentration and bound toxin is known. Additionally a stochasticity has been observed in GUV experiments, wherein the fraction of leaked GUVs from several repeated experiments was found to change exponentially with time, having a distinct dependence on the toxin concentration. ${ }^{\mathbf{1 9}}$

Models which combine detailed oligomerization kinetics and pore formation of PFTs and peptides have only recently appeared in the literature and these models can potentially provide insight into the oligomerization pathways. Vaidyanathan et al. ${ }^{21}$ studied the rupture and leakage from RBCs when exposed to ClyA. Using a model which incorporated adsorption, binding, oligomerization and rupture kinetics, both sequential irreversible and nonsequential kinetics were found to yield similar predictions to the hemoglobin release dynamics. However the sequential model was found to capture the dependence of hemoglobin release on toxin concentration more accurately. In a recent modeling study by Lee et al., ${ }^{22}$ the reversible sequential model was found to yield the best predictions for pore formation kinetics of Cry1Ac, $\alpha$-hemolysin and ClyA. Using single molecule FRET based experiments the assembly of ClyA has been analyzed in detergent and the non-sequential mechanism was found to yield the best prediction of the oligomer and pore evolution kinetics. Further, in the modelling study by Lee et al., ${ }^{22}$ the FRET pore evolution data was fit to a sequential model and comparison with other kinetic pathways were not reported. We point out that the time scales for oligomerization and kinetics in detergent are typically on the order of 1000's of seconds indicating that oligomerization in detergent is significantly slower when compared with membrane driven oligomerization. In contrast, the time scales encountered in small unilamellar phospholipid vesicle leakage experiments where pore formation kinetics are probed, are typically on the order of a few minutes. In a combined AFM and electron microscopy (EM) study ${ }^{8}$ of suilysin oligomerization, oligomer distributions were described by sequential kinetics and interaction between arcs, as observed by Mulvihill et $a .^{6}$ in a time dependent AFM study of LLO oligomerization, were not observed. In a recent review of various oligomerization pathways across $\alpha$ toxins, the sequential mechanism appears to be the most prevalent. $^{23}$

In this manuscript we carry out calcein leakage experiments for the pore forming toxin ClyA on a suspension of SUVs made up of DPPC/cholesterol in a $7: 3$ molar ratio. We combine the oligomerization kinetics for ClyA with a Poisson process for pore formation in order to predict the temporal evolution of dye leakage. The model predictions were found to yield the best agreement with a reversible sequential mechanism after incorporating leakage from membrane inserted arcs ranging from 5-12 mer oligomeric intermediates. Significantly we capture both the short and long time dye release kinetics which to our knowledge has not been reported in the literature. Non-sequential mechanisms were unable to predict the release kinetics and we propose a model for pore formation and leakage based on the formation of 'pre-arcs' wherein partly oligomerized structures insert stochastically into the membrane to cause leakage.

\section{Experimental methods}

\subsection{Vesicle preparation}

Calcein loaded DPPC : cholesterol vesicles are prepared with the sonication method using the protocol described 
elsewhere. ${ }^{24}$ Briefly, DPPC (Avanti polar) and cholesterol (Sigma) are weighed to form $30 \mathrm{~mol} \%$ cholesterol membranes and dissolved in chloroform (Sigma, HPLC grade). The solution is vacuum dried and resuspended in a $70 \mathrm{mM}$ calcein solution in PBS buffer using 10 freeze-thaw cycles. Short time probe sonication for 5 minutes is used to form small unilamellar vesicles. The excess calcein is removed by passing the solution through a Sephadex G-50 column. The presence of vesicles in the eluted fractions are verified by $1 \%$ Triton-X treatment. The vesicle diameter estimated from dynamic light scattering was 60-65 nm (see ESI Fig. 1†)

\subsection{Determination of lipid concentration}

We have used the ammmonium ferrothiocyanate method to determine the lipid concentration, ${ }^{25}$ lying in the range of $0.01-$ $0.1 \mathrm{mg} \mathrm{ml}^{-1}$. The calibration curve used to determine the lipid concentration is illustrated in the ESI (Fig. 2). $\dagger$

\subsection{Fluorescence data}

An Agilent Cary Eclipse fluorescence spectrophotometer is used for the SUV leakage experiments. Calcein dye has an excitation and emission of $495 \mathrm{~nm}$ and $520 \mathrm{~nm}$ respectively. The photomultiplier (PMT) voltage was kept at $600 \mathrm{~V}$, the excitation line was at $490 \mathrm{~nm}$ whereas the emission line was at $520 \mathrm{~nm}$ with a $5 \mathrm{~nm}$ slit width. The data has been collected at intervals of $100 \mathrm{~ms}$. Experiments have been performed at a temperature of $300 \mathrm{~K}$ and in all the leakage experiments $6 \mu \mathrm{g}$ of the ClyA toxin was added to $200 \mu \mathrm{l}$ of the DPPC/cholesterol (70/30) SUV solution.

\subsection{Expression and purification of ClyA}

The ClyA encoded plasmid was obtained from J. Green (University of Sheffield, Sheffield, UK). E. coli BL21 endo ${ }^{-}$cells transformed with pPROb ClyA were grown in Terrific Broth (Pronadisa), and proteins were expressed upon induction with $500 \mu \mathrm{M}$ isopropyl thiogalactopyranoside at $16{ }^{\circ} \mathrm{C}$ for $12 \mathrm{~h}$. Proteins were purified using a procedure described earlier, ${ }^{21}$ and purified proteins were desalted in buffer containing $50 \mathrm{mM}$ Tris-HCl (pH 8.0), $5 \mathrm{mM} \beta$-mercaptoethanol, $100 \mathrm{mM} \mathrm{NaCl}$, and $10 \%$ glycerol. Cleavage of histidine tagged proteins was carried out with TEV protease to tagless proteins. The activity of ClyA was tested using RBC lysis experiments at $25{ }^{\circ} \mathrm{C}$.

\section{Modeling calcein leakage and oligomerization kinetics}

\subsection{Calcein leakage}

We briefly illustrate how the leakage data is modeled to obtain the efflux function from the experiment. If at any time $t, N_{\mathrm{l}}$ vesicles have leaked then the concentration of dye in the external solution is,

$$
C_{\mathrm{d}}=\frac{C_{\mathrm{dv}} V_{\mathrm{v}} N_{\mathrm{l}}}{V_{\mathrm{sol}}},
$$

where $C_{\mathrm{d}}$ is the external dye concentration, $C_{\mathrm{dv}}$ is the concentration of dye in the vesicles, $V_{\mathrm{v}}$ is the volume of the vesicle and
$V_{\text {sol }}$ is the volume of the bulk solution. The ratio of the volume of vesicles to the total volume of the solution is less than $2 \%$ hence we consider only the solution volume. At low dye concentration the measured fluorescence intensity (FI) varies linearly with the dye concentration.

$$
I(t)=A C_{\mathrm{d}},
$$

where $A$ is a constant. Noting that $N_{\mathrm{v}}=N_{\mathrm{u}}+N_{\mathrm{l}}$ eqn (2) can be expressed as,

$$
I(t)=B\left[1-\frac{N_{\mathrm{u}}}{N_{\mathrm{v}}}\right],
$$

where $B=A C_{\mathrm{dv}} V_{\mathrm{v}} N_{\mathrm{v}} / V_{\text {sol }}$ is a constant for a given experiment. From the measured intensity we define a normalized efflux function,

$$
F(t)=\frac{I(t)-I_{\mathrm{o}}}{I_{\infty}-I_{\mathrm{o}}}
$$

where $I_{\mathrm{o}}$ is the initial FI and $I_{\infty}$ is the final FI. Since a non-zero initial background intensity, $I_{\mathrm{O}}$ is always present in these experiments we modify eqn (3) to yield,

$$
I(t)=B\left[1-\frac{N_{\mathrm{u}}}{N_{\mathrm{v}}}\right]+I_{\mathrm{o}} .
$$

Substituting eqn (5) into (4) we obtain

$$
F(t)=\frac{N_{\mathrm{v}}-N_{\mathrm{u}}}{N_{\mathrm{v}}-N_{\mathrm{u}}^{\infty}}=\frac{N_{\mathrm{l}}}{N_{1}^{\infty}},
$$

where $N_{\mathrm{u}}^{\infty}$ and $N_{\mathrm{l}}^{\infty}$ are the number of unleaked and leaked vesicles at long time. From eqn (6) the measured efflux function is the fraction of leaked vesicles in the system. If one considers the fraction of unleaked vesicles, then

$$
1-F(t)=E(t)=\frac{N_{\mathrm{u}}-N_{\mathrm{u}}^{\infty}}{N_{\mathrm{v}}-N_{\mathrm{u}}^{\infty}} .
$$

\subsection{Oligomerization kinetics}

Oligomerization and pore formation proceeds with the membrane bound monomer $m$ undergoing a conformational change to convert to a protomer $p_{1}$. Subsequently the protomer undergoes further oligomerization to form higher order oligomers, $p_{i}$. We investigate both sequential and non-sequential kinetic mechanisms for oligomerization and pore formation as illustrated in Fig. 1. In irreversible sequential kinetics (IRS), oligomerization occurs sequentially by the addition of a protomer to other protomers or oligomers. In the case of reversible sequential kinetics, with the exception of the conformational change, all the steps are considered reversible. The model is developed under the assumption that the forward and backward rate constants are similar for all the different kinetic mechanisms examined. This assumption significantly reduces the parameters, and has also been shown to effectively predict oligomerization and leakage kinetics in other modelling studies. ${ }^{21,22}$ 
a)

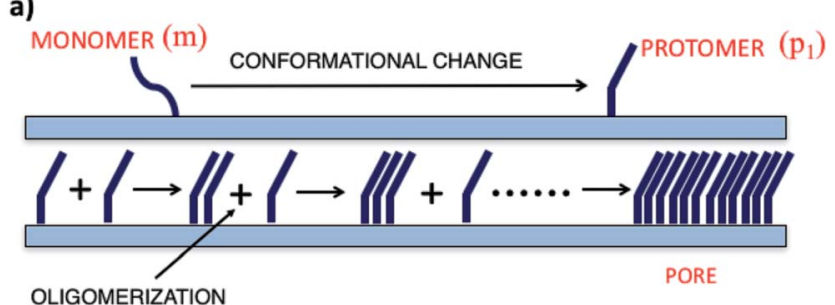

OLIGOMERIZATION

b) Reversible Sequential Mechanism

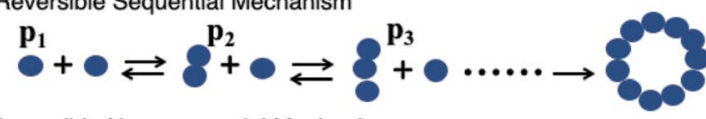

c) Reversible Non-sequential Mechanism

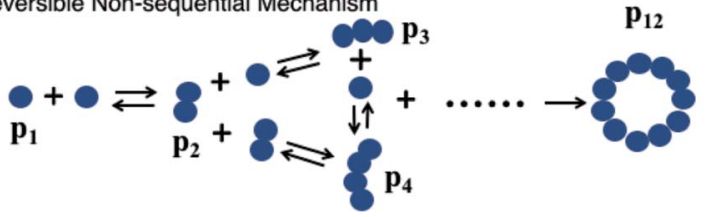

Fig. 1 Schematic illustrating the pore formation process. (a) The membrane bound monomer first transforms to a protomer and subsequent membrane mediated oligomerization results in the formation of the pore complex. (b) Protomers assemble in a reversible sequential mechanism to form the dodecameric pore complex $p_{12}$. (c) Assembly via a reversible non-sequential mechanism where we illustrate the formation of a $p_{4}$ intermediate (arc) by two different pathways.

The mass balance on the monomer is,

$$
\frac{\mathrm{d} m^{*}}{\mathrm{~d} \tau}=-\alpha_{\mathrm{c}} m^{*}
$$

and the mass balance for the different oligomers are

$$
\frac{\mathrm{d} p_{1}^{*}}{\mathrm{~d} \tau}=-p_{1}^{*} \sum_{j=1}^{11} p_{j}^{*}+\alpha_{\mathrm{b}} \sum_{j=2}^{12} p_{j}^{*}\left(1+\delta_{2, i}\right)+\alpha_{\mathrm{c}} m^{*}, \quad i=1,3,5 \ldots 11
$$

and

$$
\begin{aligned}
\frac{\mathrm{d} p_{i}^{*}}{\mathrm{~d} \tau} & =p_{1}^{*}\left[\left(p_{i-1}^{*}\left(1-\frac{\delta_{1, i-1}}{2}\right)\right)-p_{i}^{*}\left(1-\delta_{12, i}\right)\right]-\alpha_{\mathrm{b}}\left(p_{i}^{*}-p_{i+1}^{*}\right) . \\
i & =2,3,4 \ldots 12
\end{aligned}
$$

In the above equations the dimensionless variables are,

$$
m^{*}=\frac{m}{m_{0}}, \quad p_{i}^{*}=\frac{p_{i}}{m_{0}}, \tau=t k_{\mathrm{f}} \mathrm{m}_{0}, \quad \alpha_{\mathrm{c}}=\frac{k_{\mathrm{c}}}{k_{\mathrm{f}} m_{0}}, \quad \alpha_{\mathrm{b}}=\frac{k_{\mathrm{b}}}{k_{\mathrm{f}} m_{0}}
$$

where $m$ is the membrane bound monomer concentration, $m_{0}$ is the reference monomer concentration, $k_{\mathrm{f}}$ is the forward rate constant and $k_{\mathrm{c}}$ is the conformational rate constant. In eqn (8), the Kronecker delta, $\delta_{i, j}=0$ when $i \neq j$ and $\delta_{i, j}=1$ when $i=j$. We further define the following time constants; forward reaction or oligomerization time, $\tau_{\mathrm{f}}=1 / k_{\mathrm{f}} m_{0}$, the backward reaction time $\tau_{\mathrm{b}}$ $=1 / k_{\mathrm{b}}$ and the conformational time, $\tau_{\mathrm{c}}=1 / k_{\mathrm{c}}$. Hence $\alpha_{\mathrm{c}}=\tau_{\mathrm{f}} / \tau_{\mathrm{c}}$ and $\alpha_{\mathrm{b}}=\tau_{\mathrm{f}} / \tau_{\mathrm{b}}$. In case of irreversible kinetics $\alpha_{\mathrm{b}}=0$ in the above equations. In the above formulation we have assumed that the initial membrane binding is fast (seconds) when compared with the time scales for membrane assisted oligomerization which typically occurs on time scales greater than a few minutes. ${ }^{21} \mathrm{~A}$ complete analysis which includes membrane binding kinetics of the ClyA monomer coupled with oligomerization kinetics has been used in our earlier study of RBC lysis dynamics. ${ }^{21}$

In the non-sequential (NS) mechanisms, higher order oligomers can be formed not only by the addition of a single protomer but via various allowed integer combinations e.g. a tetramer can be formed by the addition of two dimers as well as the addition of a trimer to a protomer. All these steps can be either reversible or irreversible. In case of the NS mechanism oligomerization is assumed to proceed via bimolecular association or dissociation steps resulting in second order kinetics. Hence a tetramer cannot be formed by the addition of 2 protomers and one dimer. The mass balance equations for the NS mechanism are given below,

$$
\frac{\mathrm{d} m^{*}}{\mathrm{~d} \tau}=-\alpha_{\mathrm{c}} m^{*}
$$

$$
\begin{aligned}
\frac{\mathrm{d} p_{i}^{*}}{\mathrm{~d} \tau}= & \frac{i-1}{2} \sum_{j=1}^{*} p_{j}^{*} p_{i-j}^{*}+\alpha_{\mathrm{b}} \sum_{j>1}^{12} p_{j}^{*}\left(1+\delta_{2 i, j}\right)-\frac{i-1}{2} \alpha_{\mathrm{b}} p_{i}^{*}-\sum_{j=1}^{12-i} p_{i}^{*} p_{j}^{*} \\
& +\alpha_{\mathrm{c}} m^{*} \delta_{i, 1}, \\
i= & 1, \quad 3, \quad 5 \ldots 11
\end{aligned}
$$

$$
\begin{aligned}
\frac{\mathrm{d} p_{i}^{*}}{\mathrm{~d} \tau}= & \sum_{j=1}^{\frac{i}{2}} p_{j}^{*} p_{i-j}^{*}\left(1-\frac{\delta_{j, i-j}}{2}\right)+\alpha_{\mathrm{b}} \sum_{j>1}^{12} p_{j}^{*}\left(1+\delta_{2 i, j}\right)-\frac{i}{2} \alpha_{\mathrm{b}} p_{i}^{*} \\
& -\sum_{j=1}^{12-i} p_{i}^{*} p_{j}^{*} \\
i= & 2, \quad 4, \quad 6 \ldots 12
\end{aligned}
$$

The RNS mechanism is recovered by setting $\alpha_{\mathrm{b}}=0$ in the above equations.

\subsection{Leakage function and Poisson statistics}

Calcein leakage is treated as a Poisson process, wherein we assume that the arrival times (due to the formation) of leaky intermediates/arcs or pores on the vesicles can be modeled using a Poisson distribution. ${ }^{26}$ Since the number of vesicles, $N_{\mathrm{v}}$ is significantly larger than the number of protein molecules available for pore formation this assumption is justified. We point out that the diffusion time for the calcein molecules to leak out through a pore or arc, can been estimated using a simple diffusion model which results in the relation, $t_{\mathrm{D}}=V_{\mathrm{v}} l_{\mathrm{p}} /$ $A_{\mathrm{p}} D$, where $t_{\mathrm{D}}$ is the diffusion time for calcein leakage, $V_{\mathrm{v}}$ is the vesicle volume, $l_{\mathrm{p}}=10 \mathrm{~nm}$ is the length of the ClyA pore, $A_{\mathrm{p}}$ is the effective pore or arc area and $D=100 \mu^{2} \mathrm{~s}^{-1}$ is the diffusion constant of calcein. The time scales for leakage are on the order of milliseconds irrespective of whether we consider leakage through an arc or through a full pore. We obtain a leakage time of $3.6 \mathrm{~ms}$, through a dodecameric ClyA pore of 
radius $1.25 \mathrm{~nm}$ for a vesicle of diameter $70 \mathrm{~nm}$. Since these diffusion time scales through the arcs/pores are significantly smaller compared to the conformational and oligomerization time scales, the dye leakage mechanism is controlled by pore formation time scales which are on the order of seconds to minutes. Therefore we do not consider leakage times from the different sized arcs or pores. As a consequence, the measured time variation of the fluorescence intensity reflects the rate of pore formation on the vesicle. This separation of time scales is an important factor while analyzing leakage data from SUVs. ${ }^{26}$ Typical estimates for $N_{\mathrm{v}}$ lie in the range of 1.5-1.9 $\times 10^{13}$ and the protein to lipid ratios range from $30-35$ protein molecules/ vesicle. Since the ClyA pore is dodecameric, ${ }^{27}$ the small number of protein molecules per vesicle indicate that several vesicles are likely to have partially formed oligomers. We shall see that incorporating leakage from these partially formed oligomers plays an important role while making contact with the calcein leakage data.

If $\lambda_{i}$ represents the average rate of formation of an oligomer containing $i$ mers $\left(p_{i}\right)$ on the vesicle surface then the probability that a vesicle contains $k$ such pores is,

$$
E\left(k ; \lambda_{i}, t\right)=\frac{\left(\lambda_{i} t\right)^{k}}{k !} \mathrm{e}^{-\lambda_{i} t}
$$

The probability that a vesicle will not have any pores is obtained by setting $k=0$ in eqn (10),

$$
E\left(k=0 ; \lambda_{i}, t\right)=\mathrm{e}^{-\lambda_{i} t} .
$$

If $N_{\mathrm{u}}$ represents the number of unleaked vesicles, $N_{\mathrm{l}}$ the number of leaked vesicles and $N_{\mathrm{v}}$ the total number of vesicles in the system, then the probability of observing $N_{\mathrm{u}}$ unleaked vesicles is, $N_{\mathrm{u}} / N_{\mathrm{v}}$ and

$$
N_{\mathrm{u}} / N_{\mathrm{v}}=\mathrm{e}^{-\lambda_{i} t}
$$

During a leakage experiment not all the vesicles leak. If $N_{\mathrm{u}}^{\infty}$ is the number of unleaked vesicles at long time then,

$$
\frac{N_{\mathrm{u}}-N_{\mathrm{u}}^{\infty}}{N_{\mathrm{v}}-N_{\mathrm{u}}^{\infty}}=\mathrm{e}^{-\lambda_{i} t} .
$$

In the above analysis we assume that leakage occurs through a well defined pore with a fixed number of mers. In the case of ClyA it is natural to assume that leakage would occur through a dodecameric pore, however it is possible that leakage could occur through partially formed intermediates or arcs.

In order to incorporate leakage through smaller intermediates or arcs, we assume that leakage occurs independently from the different oligomeric intermediates. We propose a pre-arc based model to support this hypothesis later in the text. In this situation leakage is assumed to occur through independent Poisson processes since leakage occurs instantaneously once a pore is formed on a particular vesicle. In this scenario, the probability of observing $k$ and $l$ pore intermediates due to independent pore formation rates $\lambda_{i}$ and $\lambda_{j}$ are,

$$
E\left(k, l ; \lambda_{i}, \lambda_{j}, t\right)=\frac{\left(\lambda_{i} t\right)^{k}}{k !} \frac{\left(\lambda_{j} t\right)^{l}}{l !} \mathrm{e}^{-\left(\lambda_{i}+\lambda_{j}\right) t} .
$$

The probability of not observing either $k$ or $l$ intermediates is obtained by setting $k=0$ and $l=0$, resulting in

$$
E\left(k=0, l=0 ; \lambda_{i}, \lambda_{j}, t\right)=\mathrm{e}^{-\left(\lambda_{i}+\lambda_{j}\right) t} .
$$

Extending this, for $n$ independent rates corresponding to $n$ distinct pore intermediates, then the probability of not observing any leakage is simply an extension of eqn (15) giving rise to the leakage or efflux function,

$$
E(t)=\exp \left(-\sum_{i} \lambda_{i} t\right)
$$

where the summation over $i$ will represent the number of independent rate processes in the system. We will see that our model predictions are optimal when we consider leakage from oligomeric intermediates ranging from $p_{5}$ to $p_{12}$. Since $\lambda_{i}$ 's in the Poisson process represent the average arrival rates of the pores on the vesicle surface,

$$
\lambda_{i}=\frac{1}{t} \int_{0}^{t} \frac{\mathrm{d} p_{i}}{\mathrm{~d} t^{\prime}} \mathrm{d} t^{\prime} \quad i=1 \ldots 12
$$

Substituting the expression for $\lambda_{i}$ from eqn (17) into (16) we obtain,

$$
E(t)=\exp \left(-\sum_{i} p_{i}\right)
$$

where $p_{i}(t)$ is the time evolution of the pore population on the vesicle surface obtained by integrating the rate expressions for a given oligomerization mechanism. Hence eqn (18) connects the pore formation kinetics to the efflux function $E(t)$ determined experimentally. The observed leakage data is fit to the following efflux function,

$$
E(t)=\exp \left(-\gamma \sum_{j=n}^{12} p_{j}^{*}\right)
$$

where $\gamma$ is the number of protein molecules on a per vesicle basis and $n$ is the lower limit of the smallest $n$-mer transmembrane complex that contributes to leakage.

For a given mechanism, we seek a solution to the oligomerization equation (eqn (8) or (9)) coupled with eqn (19). The unknown parameters are $\gamma, \tau_{\mathrm{f}}, \alpha_{\mathrm{b}}$ and $\alpha_{\mathrm{c}}$. We have estimated the lipid concentration to range from $1-1.3 \mathrm{mg} \mathrm{ml}^{-1}$ using the procedure described earlier and this concentration is used to estimate the number of vesicles which lie in the range of 1.5$1.9 \times 10^{13}$. The protein concentration used in the experiment is $0.68 \mu \mathrm{M}$ and the solution volume is $260 \mu \mathrm{l}$. Using a mean vesicle size of between $65-70 \mathrm{~nm}$ and a mean molecular area for the lipid (DPPC) as $70 \AA^{2}$ we estimate $\gamma$ to lie between 30-35. Although we have a good estimate for the range for $\gamma$ values, we treat $\gamma$ as a free parameter and check the sensitivity of the model predictions to $\gamma$. We obtain estimates for $\tau_{\mathrm{f}}$ from double 
exponential fits to the calcein leakage data which are used as initial guesses for the optimization routines. This leaves $\alpha_{\mathrm{c}}$ and $\alpha_{\mathrm{b}}$ as the primary unknown parameters at a fixed value of the number of $n$-mer complexes that can contribute to leakage. The optimal parameter set is obtained using a genetic algorithm technique, using the function GA, in MATLAB 2013a. This technique can be used for optimization in the absence of a well defined range for the model parameters. This technique does not require a continuous functional form or objective function and the optimized parameters are passed to subsequent generations while seeking the optimal solution.

\section{Results and discussion}

\subsection{Leakage data}

Fig. 2 illustrates the normalized efflux function, $E(t)$ for the intensity changes during the calcein leakage experiments from vesicles incubated with ClyA. The efflux function for the dye shows the characteristic fast and slow time scales typically observed in SUV leakage experiments. In order to estimate the time constants associated with these regimes we use a double exponential fit,

$$
E(t)=A \exp \left(-t / \tau_{1}\right)+B \exp \left(-t / \tau_{2}\right)
$$

where $\tau_{1}$ and $\tau_{2}$ are the two time constants and $A$ and $B$ are arbitrary weights. We obtain a fast time constant of 0.45 minutes and a longer time constant of 5.68 minutes (Table 1) upon averaging data from four independent experiments. The fast time constant corresponds to the time required for binding, conformational change, oligomerization and initial pore formation. Binding is usually assumed to be a fast process ${ }^{\mathbf{1 8}}$ typically on the order of a few ms, hence the long time constant is related to the time scale for pore formation. The double exponential fit given in eqn (20) is primarily used to obtain estimates of the time constants which can be compared with the

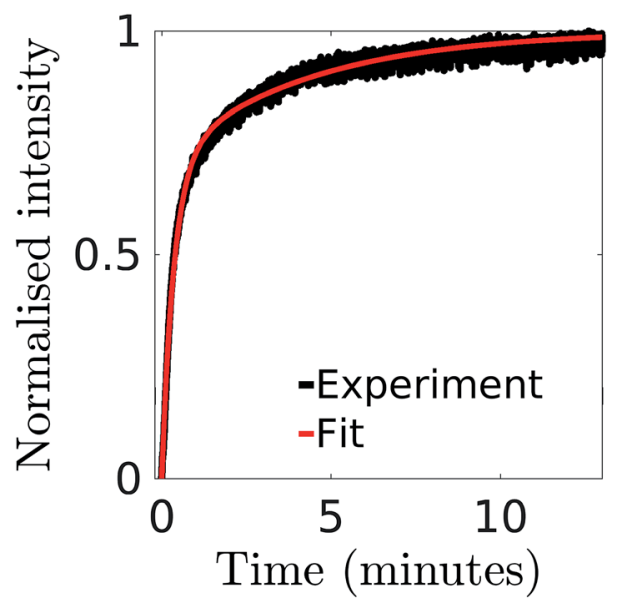

Fig. 2 Normalized intensity for calcein leakage from vesicles where 6 $\mu \mathrm{g}$ of ClyA has been incubated with $200 \mu$ solution of DPPC : chol (7:3) SUVs. The data is fitted to a double exponential function (eqn (20)) and the parameters $\tau_{1}$ and $\tau_{2}$ which correspond to the fast and slow time constants are given in Table 1.
Table 1 Parameters from the double exponential (eqn (20)) fit to the leakage data where where $A$ and $B$ are arbitrary constants that weight the fast and slow time constants $\tau_{1}$ and $\tau_{2}$ respectively. Two distinct time constants are observed

\begin{tabular}{lc}
\hline Model parameters & Mean values \\
\hline$A$ & $0.79 \pm 0.074$ \\
$\tau_{1}(\min )$ & $0.45 \pm 0.162$ \\
$\tau_{2}(\min )$ & $5.68 \pm 1.893$ \\
$B$ & $0.21 \pm 0.074$
\end{tabular}

parameters obtained from a detailed model which combines oligomerization kinetics of pore formation with a Poisson model for leakage. The presence of the slow time constant indicates that pore formation and consequently leakage can also take place on the order of a few minutes. We discuss this aspect in more detail later in the text.

\subsection{Model predictions}

Optimized or best fit parameters to the efflux function $E(t)$ are obtained for the irreversible sequential (IRS), reversible sequential (RS), reversible non-sequential (RNS) and irreversible non-sequential (IRNS) mechanisms. In case of the irreversible mechanisms three parameters, $\alpha_{\mathrm{c}}, \tau_{\mathrm{f}}$ and $\gamma$ are optimized, and for the reversible mechanism, in addition to these parameters, $\alpha_{\mathrm{b}}$ is also optimized. We have optimized these parameters to obtain the best fit, for different mechanisms by considering leakage contributions from various oligomer fractions in the efflux function. The optimized parameters for the RS mechanism are given in Table 2 and parameters for the other mechanisms are in the ESI (Tables 1-3). $\uparrow$ The predicted efflux functions upon considering various populations of the oligomers that contribute to leakage are illustrated in Fig. 3 and 4 for the non-sequential and sequential mechanisms respectively. The results from different mechanisms are discussed next.

4.2.1 Non-sequential oligomerization. For both the IRNS and RNS oligomerization mechanisms (Fig. 3), the model is unable to capture trends observed in the ClyA leakage data, and the temporal evolution predicted by the models are relatively insensitive to the different oligomer populations that contribute

Table 2 The optimized parameters for the reversible sequential (RS) mechanism as a function of various mer fractions that contribute to leakage. $\alpha_{c}=\tau_{f} / \tau_{c}$, the ratio of forward to conformational time constant and $\alpha_{\mathrm{b}}=\tau_{\mathrm{f}} / \tau_{\mathrm{b}}$, the ratio of forward to backward time constant. A distinct increase in the value of $\gamma$ is observed as the contribution from the smaller mers is reduced. The best agreement with the calcein leakage data is obtained for the 5-12 mer fraction

\begin{tabular}{llrll}
\hline Oligomers & $\alpha_{\mathrm{b}}$ & $\alpha_{\mathrm{c}}$ & $\tau_{\mathrm{f}}(\mathrm{s})$ & $\begin{array}{l}\text { Proteins per } \\
\text { vesicle }(\gamma)\end{array}$ \\
\hline $5-12$ & 0.026 & 5.9 & 4.4 & 35 \\
$7-12$ & 1.3 & 2.0 & 3.0 & 54 \\
$9-12$ & 0.28 & 12.3 & 2.9 & 90 \\
12 & 0.17 & 6.8 & 2.5 & 227
\end{tabular}



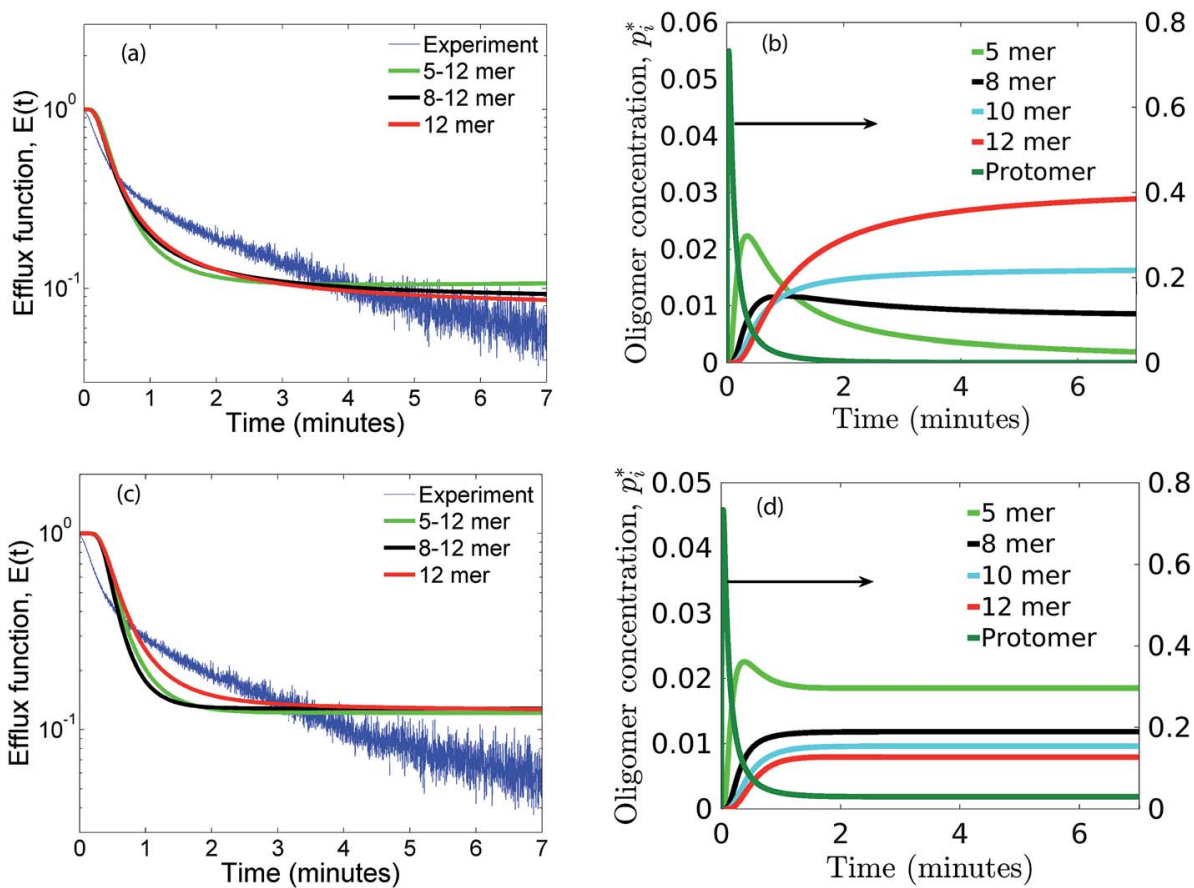

Fig. 3 Model predictions for (a) irreversible non sequential (IRNS) and (c) reversible non sequential (RNS) mechanisms, for various oligomer populations included in the efflux function. The NS mechanism does not predict the time evolution of calcein leakage. (b) and (d) are the evolution of various oligomers corresponding to the optimized parameters for the 5-12 mer population for the IRNS and RNS populations respectively.
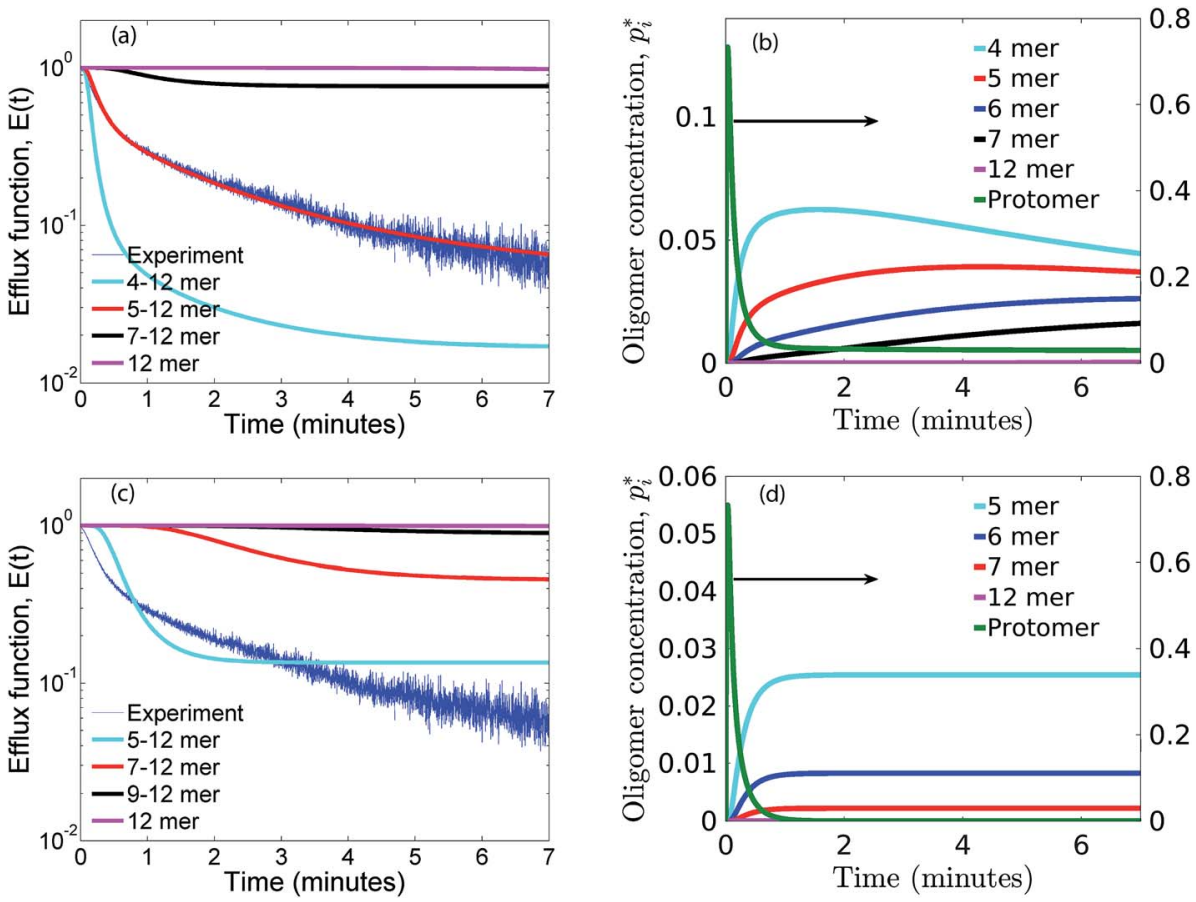

Fig. 4 Model predictions for (a) reversible sequential (RS) and (c) irreversible sequential (IRS) mechanisms, for various mer populations in the efflux function. The IRS mechanism is unable to predict the leakage data, whereas excellent predictions are obtained for the RS mechanism, when mer populations in the range 5-12 contribute to leakage. (b) and (d) depict the time evolution of oligomers for optimized parameters corresponding to the 5-12 mer populations for RS and IRS mechanisms respectively. 
to leakage. The optimized parameter sets for the IRNS mechanism are given in the ESI (Tables 1 and 2) $\dagger$ where the values of $\gamma$ increase monotonically when arc populations contributing to leakage are reduced. Hence the smallest value of $\gamma$ is observed for the 5-12 mer arc populations. Noting that the estimated value of $\gamma$ lies between 30-35 proteins per vesicle we obtain much larger values when the arc populations are reduced. In the IRNS mechanism the oligomer populations (Fig. 3b) achieve steady states at longer times, as this is dependent on the depletion of protomers, $p_{1}$ and lower order mers from the vesicle surface. However in the RNS mechanism, steady states are achieved at shorter times and a more uniform distribution of oligomer populations are observed. As a consequence the efflux function for the RNS mechanism (Fig. 3c) has a well defined steplike behavior when compared with the efflux function from the IRNS mechanism (Fig. 3a). Due to reversibility, the values of $\gamma$ are significantly higher $(50-227)$ to compensate for the lower fraction of higher order oligomer populations.

4.2.2 Sequential oligomerization. We illustrate the comparison between the model and experimental data for the RS mechanism in Fig. 4a. In this model larger oligomers form only by sequential addition of the protomer $\left(p_{1}\right)$. We have included reversibility for all the higher order mers including the 12 mer. The optimized parameters for this case are given in Table 2 . The best agreement was obtained for arc populations varying between 5-12 mers and the influence of the model predictions as a function of the mer fractions as well as the parameters $\alpha_{\mathrm{b}}$ and $\alpha_{\mathrm{c}}$ will be discussed later in the text. For $\alpha_{\mathrm{c}}=5.9$ and $\tau_{\mathrm{f}}=4.4 \mathrm{~s}$ the conformational time constant, $\tau_{\mathrm{c}}=0.745 \mathrm{~s}$. The protein to vesicle ratio is $\gamma=35$ and $\alpha_{\mathrm{b}}=0.026$ results in a value of $\tau_{\mathrm{b}}=169.2 \mathrm{~s}$ for the backward time constant indicating a significantly slower reverse kinetics. Using these parameters, the model predictions are in excellent agreement with the observed efflux function, $E(t)$ as illustrated in Fig. 4a. We also point out that the data are shown in a semi-log scale where changes at the smaller values of the intensity function are amplified. The accuracy of the fit across the entire observation time is evident. In the Poisson process we have included different ranges of oligomers $p_{i}$ which contributes to leakage. Interestingly the model is able to capture both the short and long time release dynamics extremely accurately. We point out that earlier predictions of leakage data based on the Poisson model, were only able to capture the initial linear pore formation kinetics ${ }^{18,28}$ by assuming that the oligomeric pore complex was solely responsible for pore formation and leakage. The slower and longer time component was not captured in these studies. Our results indicate that in order to capture both the short and long time release kinetics it is necessary to include leakage from a distribution of oligomers or arcs, indicating that intermediate oligomers have a dominant contribution to leakage.

Fig. $4 \mathrm{~b}$ illustrates the time evolution of oligomers for parameter values obtained for the best fit to the leakage data. The key observation is the rapid rise in the lower order oligomers at the early times. The data in Fig. $4 \mathrm{~b}$ corresponds to the optimal solution to the data shown in Fig. 4a. Here the lower order oligomers between 5, 6 and to some extent 7 show an increase between 0-0.5 minutes which contributes to the rapid drop in the efflux function during this time scale. At longer times, between
1-3 minutes, the fraction of higher order oligomers gradually increases and the rate of formation of the lower order oligomers remain steady. Over the time scale of the experiment, contributions from higher order oligomers are not significant. This is a consequence of the low protein to lipid ratios, $\gamma$ in the system. The concentration of protomers $\left(p_{1}\right)$ drops sharply and attains a steady non-zero value due to the reversibility in the kinetic model. In order to study the contribution from the higher order oligomers we obtained optimized solutions by varying the upper limit of the mers that contribute to leakage (ESI Fig. $3 \dagger$ ). The leakage function is significantly underestimated if only 5 mers are assumed to contribute to leakage. Including 5-7 mers captures the short time transient and is able to predict the leakage function up to 1 minute. However the longer time leakage response is underestimated at these oligomer populations. The predictions from 5-10 mers improves the long time (1-7 minutes) contribution to the leakage function and excellent predictions are obtained across the entire observation time. Addition of 11 and 12 mers, marginally improves the leakage predictions at longer times, indicating that the dominant contribution to leakage arises from the intermediate pores formed from $p_{5}-p_{10}$. For the estimated values of $\gamma$ and noting that ClyA forms a dodecameric pore, 2-3 pores can form per vesicle if all the protein converts to form the dodecameric pore complex.

The predictions with the IRS mechanism are illustrated in Fig. 4c, where the model is unable to capture the leakage trends. Unlike the NS mechanism the leakage is a strong function of the mer populations used in the model. Inclusion of only the higher order oligomers results in a very low leakage intensity and the corresponding $\gamma$ values (Table 2) are unusually high. This trend is observed for the RS mechanism as well. Since steady states are achieved once the $p_{1}$ population is exhausted, a steady mer distribution is observed after 2 minutes for the 5-12 mer simulation. This results in a characteristic step-like leakage function (Fig. 4c) under these conditions.

4.2.3 Oligomer distributions. The distribution of the oligomer populations at steady state, corresponding to the optimal parameter sets are illustrated in Fig. 5. Each mechanism evolves to a unique distribution of oligomers. In the irreversible mechanisms (Fig. 5a and c), a distinct feature is the absence of the protomer, $p_{1}$. In the IRNS mechanism, the steady state population evolves toward a distribution of oligomers which can no longer undergo further oligomerization to form higher order oligomers. In the reversible mechanisms (Fig. $5 \mathrm{~b}$ and $\mathrm{d}$ ), a constant supply of $p_{1}$ is present, and the distributions show a high population of lower order oligomers. The extent of formation of the lower order oligomers is dictated by the extent of reversibility in the model. Additionally the time taken to reach steady state is significantly longer than the time duration of the leakage data, indicating that leakage as observed in the experiments is dominated by a transient state of pore formation on the vesicle.

\subsection{Assessing reversibility; $\alpha_{\mathrm{b}}$}

The evolution of the efflux function, with varying values of $\alpha_{\mathrm{b}}$ are illustrated in Fig. 6a for the RS mechanism. For a wide range of $\alpha_{\mathrm{b}}$, which is the ratio of the forward to backward time 

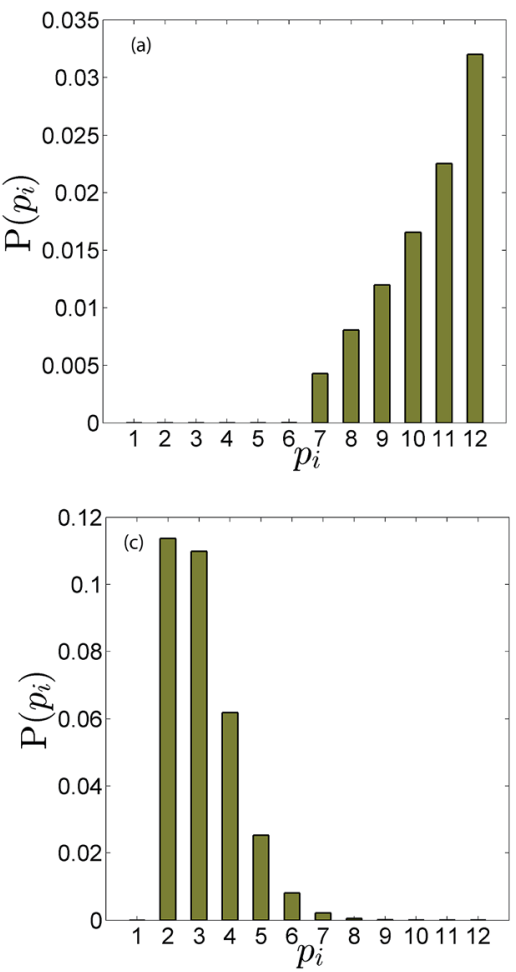
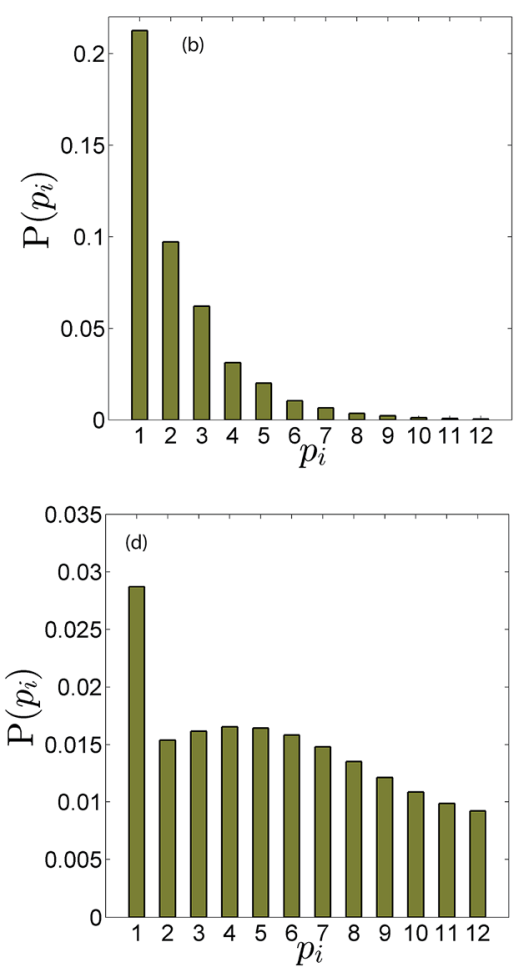

Fig. 5 Steady state distribution of various oligomers for (a) irreversible non-sequential, IRNS, (b) reversible non-sequential RNS, (c) irreversible sequential IRS and (d) reversible sequential RS based on the optimized parameters obtained while considering leakage from the 5-12 mer populations.

constants, the initial fast time constant in the leakage data is captured quite accurately, however the values of $\alpha_{\mathrm{b}}$ is seen to have a strong influence on the longer time evolution of the efflux function and the entire temporal evolution is captured only when $\alpha_{\mathrm{b}}$ lies in the range of $0.02-0.03$. As $\alpha_{\mathrm{b}}$ is lowered, the mechanism tends to that of the IRS mechanism. In this mechanism oligomerization ceases when the protomer concentration $p_{1}$ reduces to zero. Hence a steady distribution of the lower oligomers which dominate the early dynamics, are achieved at earlier times for higher values of $\alpha_{\mathrm{b}}=0.2$ (Fig. 6b) leading to a relatively constant efflux evolution at earlier times when compared with the prediction at lower values of $\alpha_{\mathrm{b}}=$ 0.002 (Fig. 6c). At intermediate values of $\alpha_{\mathrm{b}}$ (Fig. 6b) the 5 mer population is seen to go through an initial maximum. The extent of reversibility plays an important role in the evolution of the population of mers on the surface of the membrane. In the absence of reversibility, oligomerization ceases when $p_{1}$ concentration drops to zero for the sequential mechanisms. However even a small amount of reversibility maintains a supply of $p_{1}$ which influences the overall oligomer evolution. The steady distribution of oligomers in the case of the RS mechanism is more evenly distributed when compared with the distribution obtained for the IRS mechanism where the distribution is skewed toward the lower order oligomers which remain trapped once $p_{1}$ is no longer present (Fig. 5c). Our analysis indicates that reversibility plays an important role in maintaining a supply of intermediate oligomers that can contribute to leakage. This is consistent with findings in this study as well as with the oligomerization models that were used to fit pore formation kinetics by Lee et al.. ${ }^{22}$ We point out that in our analysis we have assumed that the $p_{12}$ or the ClyA pore complex can reversibly breakup to form $p_{1}$ and $p_{11}$. Relaxing this assumption did not alter the model predictions due to the low concentration of $p_{12}$ oligomers when compared with the concentration of the lower order oligomers formed for the time scales involved in the leakage experiments.

\subsection{Influence of conformation and oligomerization times, $\alpha_{c}$}

The influence of the parameter $\alpha_{\mathrm{c}}$ which is the ratio of the oligomerization time constant to the conformational time is illustrated in Fig. 7a. Only a marginal change is observed for $\alpha_{\mathrm{c}}$ values greater than 1 , indicating that the oligomer distributions are relatively insensitive to $\alpha_{\mathrm{c}}$ in this range where the conformational changes are complete. We did not observe any significant change in the leakage evolution for $\alpha_{\mathrm{c}}$ values between 1-5 and the predictions remain unchanged upon further increase in the value of $\alpha_{\mathrm{c}}$. At higher values of $\alpha_{\mathrm{c}}$, the monomer converts rapidly to form the membrane inserted protomer and a steady state is achieved more rapidly for the RS mechanism. In contrast, the model predictions are sensitive to $\alpha_{\mathrm{c}}$ below 1. As $\alpha_{\mathrm{c}}$ is lowered, the time constant for conformational change is increased compared to the oligomerization time, and the evolution of oligomer populations is delayed (Fig. 7b and c). Hence at the lower value of $\alpha_{\mathrm{c}} \leq 1$, we observed a delay ranging from 1-2 minutes, before leakage is initiated. Since the optimal value of the time constant for oligomerization 

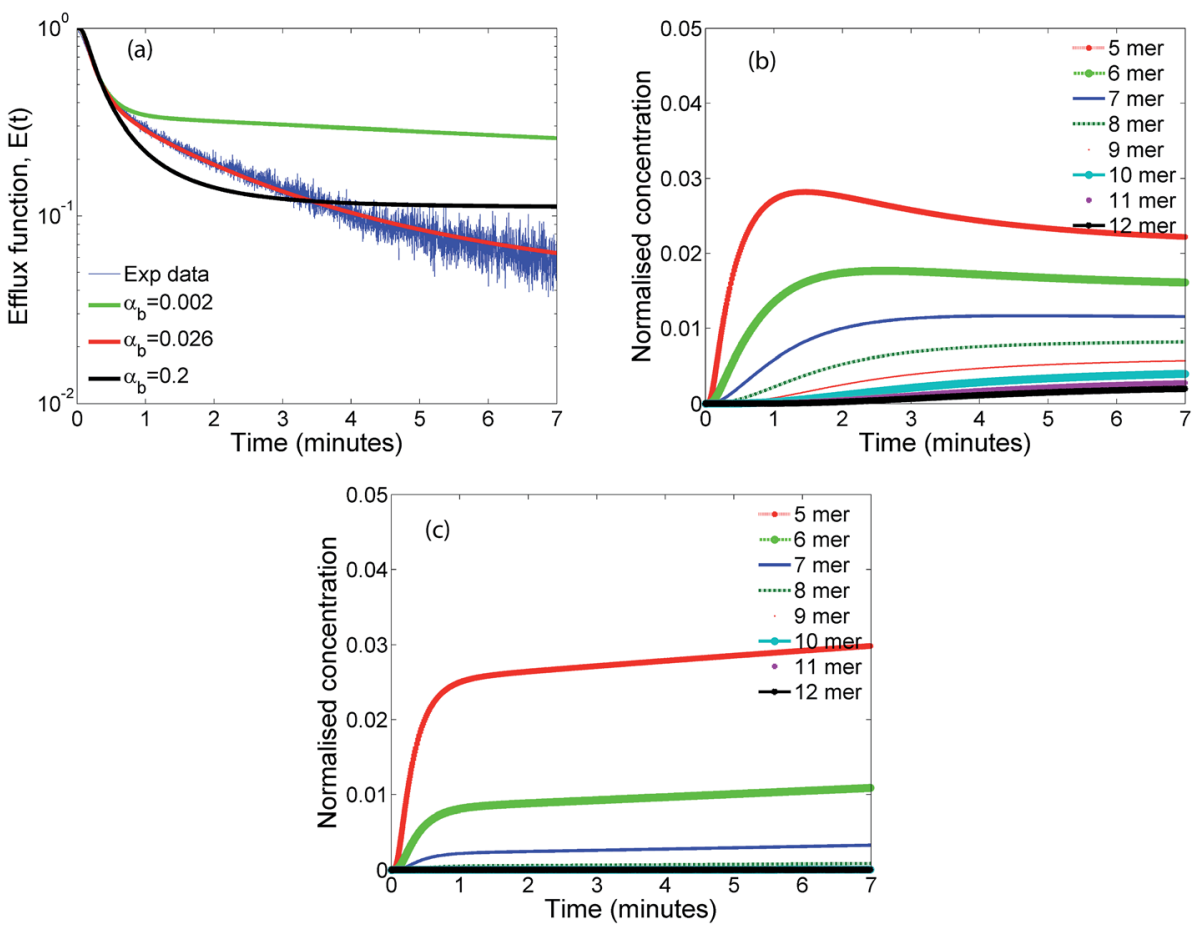

Fig. 6 Influence of $\alpha_{\mathrm{b}}=\tau_{\mathrm{f}} / \tau_{\mathrm{b}}$ on the model predictions for the RS mechanism. All values of $\alpha_{\mathrm{b}}$ are able to capture the initial leakage dynamics however for $\alpha_{\mathrm{b}}=0.026$ the entire leakage function is captured (a). Evolution of mers for $\alpha_{\mathrm{b}}=0.02$ (b) and for $\alpha_{\mathrm{b}}=0.002$ (c).
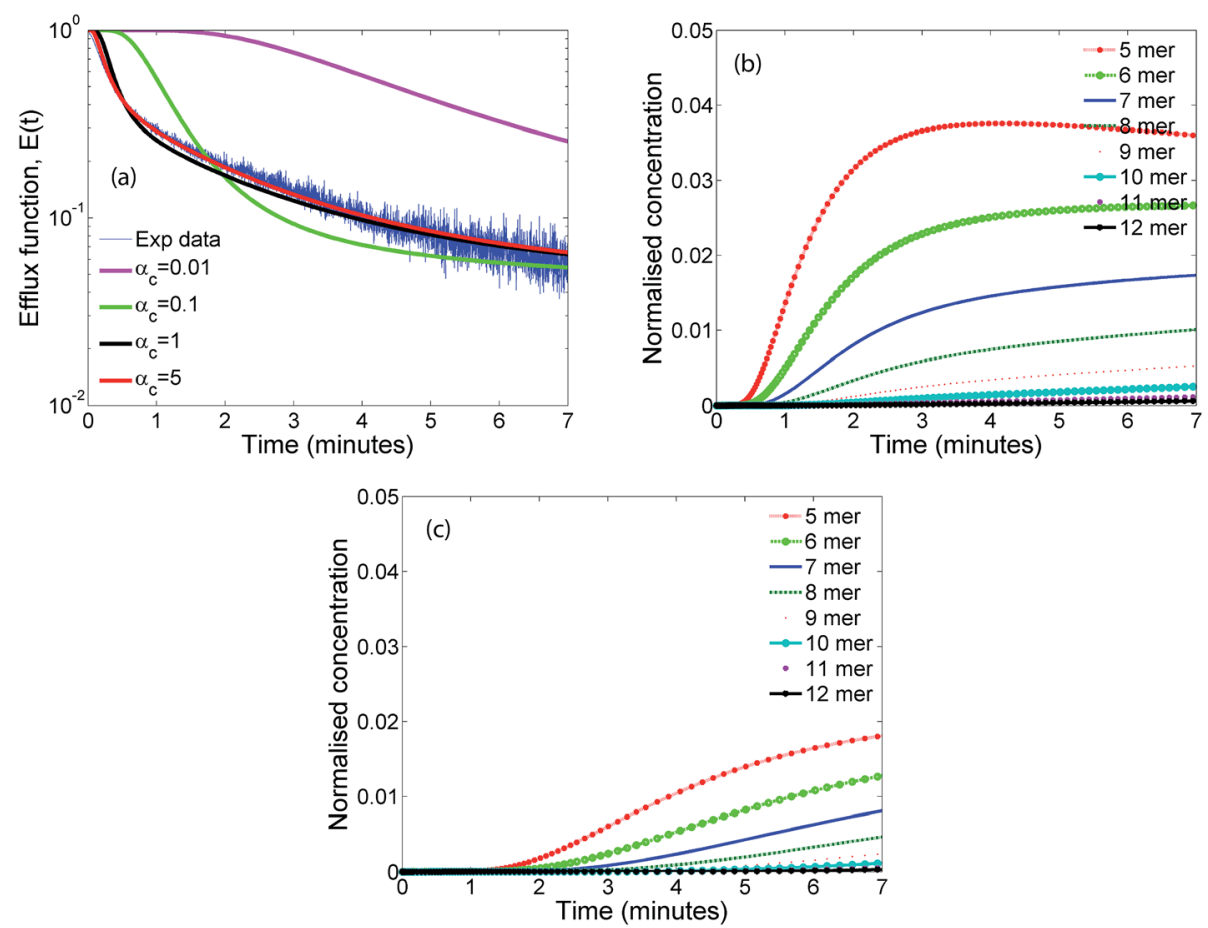

Fig. 7 Influence of $\alpha_{\mathrm{c}}$ which is the ratio of conformation to oligomerization time constants on leakage (a) and oligomer evolution (b) and (c). Lowering the value of $\alpha_{\mathrm{c}}$ results in a delay before leakage occurs due to the resulting delay in oligomer formation (b) and (c). The model predictions for leakage (a) are relatively insensitive for $\alpha_{\mathrm{c}} \geq 1 . \alpha_{\mathrm{c}}=0.1$ (b) and 0.01 (c).

$\tau_{\mathrm{f}}=1 / k_{\mathrm{f}} m_{\mathrm{o}}$ is $4.4 \mathrm{~s}$, an $\alpha_{\mathrm{c}}=\tau_{\mathrm{f}} / \tau_{\mathrm{c}}$ value of 5 and 1 imply $\tau_{\mathrm{c}}$ values of 0.88 and $4.4 \mathrm{~s}$ respectively. We point out that it is generally accepted that the conformation time is larger than the oligomerization time constant for ClyA. ${ }^{29}$ Our observation for the optimal solution indicates that the conformational time is faster or similar to the time scales for oligomerization. 
Fig. 8 illustrates the effect of varying $\gamma$ which is proportional to the protein to lipid ratio, on the model predictions. Increasing the value of $\gamma$, results in a greater drop in the efflux function due to formation of a larger number of pores. Model predictions are quite sensitive to changes in $\gamma$ and the best fit is obtained for the computed values based on the lipid and protein concentrations in the experiment which lie in the range of 30-35 proteins per vesicle.

\section{Discussion: leaky intermediates, pre-arcs and arcs}

As seen earlier, the best predictions to the efflux data are obtained while using a model that includes leakage from a range of intermediates or arc-like pore structures. The contribution from these various fractions as determined by the oligomerization kinetics influences the evolution of the efflux function. We have observed that contributions from 5 to 12 oligomers provide the best fit over the entire temporal evolution of the observed leakage. In Fig. 4a we illustrate the effect of varying the range of 'arcs' that contribute to leakage. If the only contribution to leakage is from the dodecameric 12-mer pore complex we are unable to capture the leakage data for any combination of parameter sets, since the evolution of the 12-mer complex is significantly slower when compared with the formation rates for the lower order oligomers over the time scales sampled in the experiment. Increasing the contribution from the lower order oligomers is a critical requirement in order to capture the rapid efflux at short times. Including the 4-mer population in the model results in a large overestimate of the efflux evolution. Upon considering a fully formed pore consisting of 12 protomeric units, the angle subtended by each protomeric unit is $30^{\circ}$. Based on projected areas and using a hydrodynamic radius of $0.74 \mathrm{~nm}$ for the calcein molecule we estimate the number of calcein molecules that can be filled in the arcs of ClyA. Using this geometric criterion for the pore cross-section, the 5-mer arc is able to pack about 6 calcein molecules, however for the 4-mer arc this number reduces to 4-5 calcein molecules. This simple geometric criterion only provides an upper estimate of the void space and does not account for the reduced hydrodynamic diameter of the pore due to the presence of bound water and ions. ${ }^{30}$

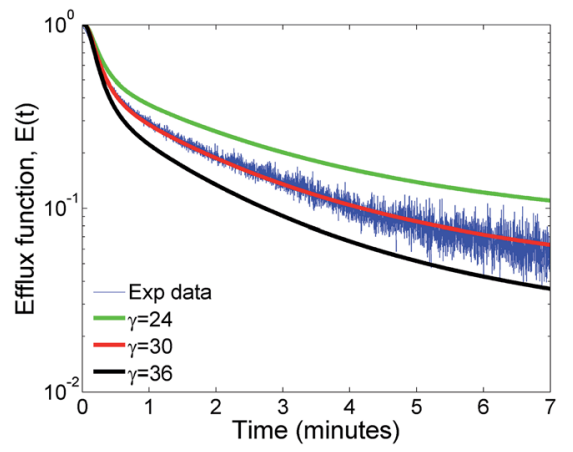

Fig. 8 Influence of the number of protein molecules per vesicle $(\gamma)$ on vesicle leakage kinetics.
The model predictions suggest that leakage from ClyA on SUVs occurs predominantly through arcs which are oligomeric intermediates formed during pore formation. The propensity to form these lower order pore oligomers or arcs that remain trapped without subsequent oligomerization is further enhanced due to the low protein to lipid ratios in our system. High curvature in SUVs could also play a role. In recent molecular dynamics simulations of ClyA, carried out in our laboratory we have shown that membrane inserted arcs or pores are lined by the protein on one face with toroidal lipids forming the pore interior in regions where proteins are absent. ${ }^{31}$ These arcs were found to be stable structures with a water channel capable of transporting material across the transmembrane channel supporting the prediction that arcs play a dominant role in the leakage. Arc-like transmembrane constructs have been purported to exist for other $\alpha$-helical transmembrane proteins such as actinoporins. ${ }^{\mathbf{1 2}}$ Since the predictions of leakage data are dominated by a population transmembrane arcs, the propensity to form the complete dodecameric pore is restricted, at least on the time scale of the leakage experiment. It is well known that curvature effects play an important role in the pore formation propensity and positive curvature lipids are known to stabilize toroidal pore formation with antimicrobial peptides. ${ }^{32}$ This supports the notion that high curvature present in the SUVs used in this study could play a role in stabilizing the formation of toroidal lipids which are required to form the transmembrane arc-like ClyA structures predicted by the model. Complete oligomerization for $\alpha$-hemolysin (a $\beta$-toxin) has been shown to be reduced in membranes with higher curvature. ${ }^{33}$

In order to rationalize leakage through these arcs we invoke the following model for pore formation as illustrated in Fig. 9. Since a higher order oligomer must necessarily be preceded by a lower order oligomer, leakage occurs through the smallest transmembrane arcs capable of transporting calcein. Noting that leakage times are significantly smaller than pore formation times, leakage would then be entirely dependent on the formation of the first leaky intermediate. Our model suggests that pore formation and leakage in the collection of SUVs is governed by the underlying stochasticity of a Poisson process. In the pre-pore model, ${ }^{\mathbf{1 0}}$ pre-arcs which are not membrane inserted, first assemble on the membrane surface (Fig. 9). Recent molecular dynamics simulations in our laboratory, reveal that this membrane inserted state is driven by the insertion of the hydrophobic $\beta$ tongue into the membrane lipids. ${ }^{11}$ At this stage the N-terminus, depicted in purple in Fig. 9 is not yet inserted into the membrane. Oligomerization takes place to form the pre-arcs $p_{i}$ and membrane insertion converts a pre-arc to an arc which is then capable of leakage. In this scenario one can envision the assembly of pre-arcs which continue to oligomerize on some vesicles to form larger pre-arcs which can eventually assemble to form the dodecameric pore provided sufficient toxin is available. Within this picture, the oligomerization kinetics as evaluated in our model, represents the rates for the formation of pre-arcs. The conversion of the pre-arc to a membrane inserted arc is assumed to be a relatively fast step when compared with the oligomerization kinetics. Since the protein to lipid ratio is small, it is highly probable that 


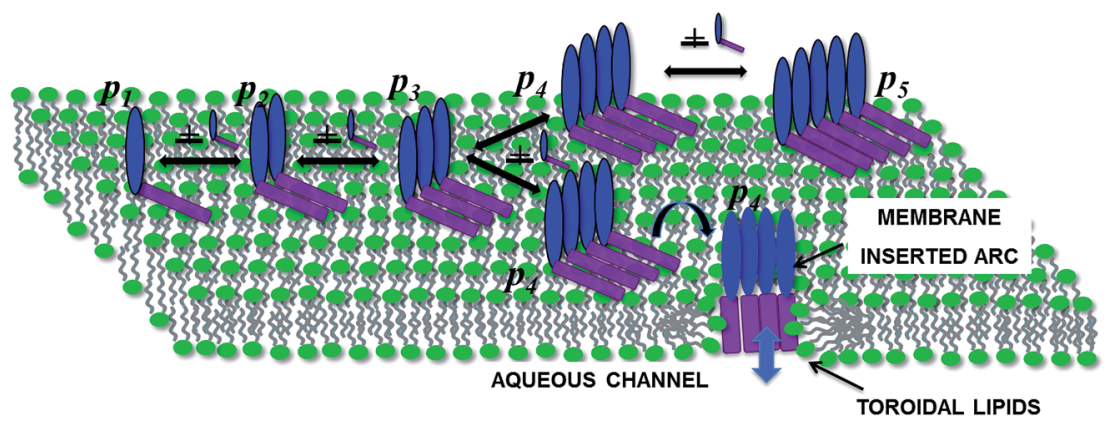

Fig. 9 Schematic illustrating the reversible sequential oligomerization pathway for ClyA. Oligomerization gives rises to pre-arcs which consist of assembled oligomeric complexes $\left(p_{i}\right)$ without having their $\mathrm{N}$-termini (purple) inserted into the membrane. Insertion to form a membrane inserted arc as shown for $p_{4}$ is stochastic and the pre-arc has the possibility of further oligomerization to form the higher order oligomer, $p_{5}$ during the course of the oligomerization pathway.

these pre-arcs can no longer oligomerize to completion and convert rapidly to a functional transmembrane arc. The model predictions further support the view that formation of transmembrane arcs capable of leakage on the membrane surface follows a Poisson process which determines the arrival times of arcs/pores on the vesicle surface.

Before concluding this section we discuss our results in light of other oligomerization mechanisms that have been proposed for ClyA as well as other cholesterol dependent cytolysins (CDCs). Our model for ClyA oligomerization on vesicles invokes certain common features associated with AFM data, albeit on CDCs. These are the presence of arcs and pre-arcs as well as the membrane inserted arcs. Note that in our model for ClyA leakage kinetics, we did not include the transition from a prearc to a membrane inserted arc and additional experimental evidence is required to elucidate the details of this mechanism, if it exists. Further, a simple sequential model was able to qualitatively match the steady state arc distributions obtained for suilysin on supported lipid membranes ${ }^{8}$ and arc-arc interactions to form pores (non-sequential mechanism) observed in a previous LLO study ${ }^{6}$ were not observed by these authors. Additional efforts are required to resolve these underlying differences. Our results concur with the reversible sequential mechanism used by Lee et al., ${ }^{22}$ to predict the kinetics of pore formation for ClyA as well as other toxins. In contrast, single molecule FRET experiments by Benke et al., ${ }^{34}$ are compatible with a non-sequential mechanism for oligomerization kinetics of ClyA in detergent where a molten globule off-pathway intermediate was found to be present in the monomer to protomer transition. It is likely that the assembly and kinetic pathways sampled for protein assembly in bulk detergent solution can vary with pathways for membrane assisted oligomerization where kinetics are about 50-100 times faster than assembly in detergent.

\section{Summary and conclusion}

We carried out calcein leakage experiments for ClyA using a suspension of SUVs made up of DPPC/Cholesterol in a $7: 3$ molar ratio. Using a Poisson model to describe the inherent stochasticity present during pore formation in the SUV suspension we test various oligomerization pathways on the vesicles by comparing the model predictions with the observed calcein leakage data for ClyA. The model predictions were found to yield the best agreement with a reversible sequential mechanism after incorporating leakage from membrane inserted arcs ranging from 5-12 mer oligomeric intermediates. The dominant contribution was found to occur from the smaller arcs, consistent with the low protein to lipid ratios used in the experiment. There are two models that have been proposed in the literature for pore formation. In the pre-pore model oligomerization proceeds on the membrane surface to form a pre-pore structure. These pre-pore complexes are not capable of leakage until they penetrate into the membrane to form a transmembrane pore complex capable of transporting ions and cellular content across the membrane. In this model the pre-pore assembly proceeds without the formation of any membrane inserted oligomers capable of leakage. In a second model known as the growing pore model, membrane inserted arcs which are partially formed oligomers are capable of leakage. Our analysis suggests a model for the pore formation mechanism of ClyA connected to the prepore hypothesis usually associated with the formation of a fully formed dodecameric pre-pore. ${ }^{10}$ However, we propose that prearcs which are oligomeric intermediates insert into the membrane in a stochastic manner to cause leakage. The primary evidence for this mechanism is derived from the reversible sequential oligomerization model which provides an excellent fit to the leakage data. In this model pre-arcs evolve and insertion and ensuing leakage occurs stochastically. This stochasticity is built into the Poisson process where the arrival times of the leaky arcs or pores follow a Poisson distribution. Further our model is able to capture for the first time both the fast and slow time constants typically observed in calcein leakage data. A key inference is that intermediate arcs play a critical role in leakage and our model predicts that leakage from arcs ranging from 5-12 mers are required to capture the entire time evolution of the leakage data. The fast time scale arises from the smaller arcs and longer slower time scales are attributed to the higher oligomeric arcs.

The insight provided by the oligomerization mechanism can be useful while developing drug targets to potentially disrupt pore formation by compromising oligomeric intermediates 
thereby preventing the formation of transmembrane channels implicated in PFT mediated infections. Our analysis in the present work has been carried out for leakage data obtained from a DPPC/Cholesterol system and it is well known that ClyA pore formation is enhanced by the presence of cholesterol. Although leakage kinetics are expected to vary with cholesterol content and the underlying transition temperature of the lipids, we expect the generic conclusions deduced in this manuscript to remain unaltered.

\section{Conflicts of interest}

There are no conflicts to declare.

\section{Acknowledgements}

This work was carried out with funding from the Department of Science and Technology (DST) under the IRHPA scheme. AA and KGA would like to thank Jaydeep Basu, Sandhya Visveshwaraiah, Sanjeev Gupta, Rahul Roy and Pradeep Sathyanarayana for several useful discussions during the course of this work. The ClyA toxins used in this work were expressed and purified by Pradeep Sathyanarayana.

\section{References}

1 M. Soskine, A. Biesemans, B. Moeyaert, S. Cheley, H. Bayley and G. Maglia, Nano Lett., 2012, 12, 4895-4900.

2 A. P. Heuck, R. K. Tweten and A. E. Johnson, J. Biol. Chem., 2003, 278, 31218-31225.

3 D. M. Czajkowsky, E. M. Hotze, Z. Shao and R. K. Tweten, EMBO J., 2004, 23, 3206-3215.

4 M. Podobnik, M. Marchioretto, M. Zanetti, A. Bavdek, M. Kisovec, M. M. Cajnko, L. Lunelli, M. Dalla Serra and G. Anderluh, Sci. Rep., 2015, 5, 1-10.

5 N. Yilmaz and T. Kobayashi, ACS Nano, 2015, 9, 7960-7967. 6 E. Mulvihill, K. van Pee, S. A. Mari, D. J. Müller and O. Yildiz, Nano Lett., 2015, 15, 6965-6973.

7 N. K. Sarangi, K. G. Ayappa, S. S. Visweswariah and J. K. Basu, Phys. Chem. Chem. Phys., 2016, 18, 29935-29945.

8 C. Leung, N. V. Dudkina, N. Lukoyanova, A. W. Hodel, I. Farabella, A. P. Pandurangan, N. Jahan, M. Pires Damaso, D. Osmanovic, C. F. Reboul, M. A. Dunstone, P. W. Andrew, R. Lonnen, M. Topf, H. R. Saibil and B. W. Hoogenboom, eLife, 2014, 3, e04247.

9 R. J. Gilbert, Biochim. Biophys. Acta, Biomembr., 2016, 1858, 487-499.

10 M. Mueller, U. Grauschopf, T. Maier, R. Glockshuber and N. Ban, Nature, 2009, 459, 726-730.
11 V. V. H. Giri Rao, R. Desikan, K. G. Ayappa and S. Gosavi, J. Phys. Chem. B, 2016, 120, 12064-12078.

12 K. Č. Kristan, G. Viero, M. Dalla Serra, P. Maček and G. Anderluh, Toxicon, 2009, 54, 1125-1134.

13 S. Qian, W. Wang, L. Yang and H. W. Huang, Proc. Natl. Acad. Sci. U. S. A., 2008, 105, 17379-17383.

14 J. M. Alam, T. Kobayashi and M. Yamazaki, Biochemistry, 2012, 51, 5160-5172.

15 M.-T. Lee, W.-C. Hung, F.-Y. Chen and H. W. Huang, Proc. Natl. Acad. Sci. U. S. A., 2008, 105, 5087-5092.

16 G. Schwarz, R.-T. Zong and T. Popescu, Biochim. Biophys. Acta, Biomembr., 1992, 1110, 97-104.

17 G. Menestrina, S. Forti and F. Gambale, Biophys. J., 1989, 55, 393-405.

18 S. Rex and G. Schwarz, Biochemistry, 1998, 37, 2336-2345.

19 Y. Tamba and M. Yamazaki, Biochemistry, 2005, 44, 1582315833.

20 Y. Tamba, H. Ariyama, V. Levadny and M. Yamazaki, J. Phys. Chem. B, 2010, 114, 12018-12026.

21 M. Vaidyanathan, P. Sathyanarayana, P. K. Maiti, S. S. Visweswariah and K. G. Ayappa, $R S C A d v ., 2014,4$, 4930-4942.

22 A. Lee, M. Senior, M. Wallace, T. Woolley and I. Griffiths, J. R. Soc., Interface, 2016, 13, 20150762.

23 K. Cosentino, U. Ros and A. J. García-Sáez, Biochim. Biophys. Acta, Biomembr., 2016, 1858, 457-466.

24 G. Menestrina, FEBS Lett., 1988, 232, 217-220.

25 J. C. M. Stewart, Anal. Biochem., 1980, 104, 10-14.

26 G. Schwarz and C. H. Robert, Biophys. J., 1990, 58, 577.

27 M. Mueller, U. Grauschopf, T. Maier, R. Glockshuber and N. Ban, Nature, 2009, 459, 726-730.

28 G. Schwarz and G. Beschiaschvili, Biochim. Biophys. Acta, Biomembr., 1989, 979, 82-90.

29 N. Eifler, M. Vetsch, M. Gregorini, P. Ringler, M. Chami, A. Philippsen, A. Fritz, S. A. Müller, R. Glockshuber, A. Engel, et al., EMBO J., 2006, 25, 2652-2661.

30 M. Taraknath, S. Kanchi, K. G. Ayappa and P. K. Maiti, Nanoscale, 2016, 8, 13045-13058.

31 R. Desikan, P. K. Maiti and K. G. Ayappa, Langmuir, 2017, 33, 11496-11510.

32 A. A. Sobko, E. A. Kotova, Y. N. Antonenko, S. D. Zakharov and W. A. Cramer, FEBS Lett., 2004, 576, 205-210.

33 S. Fujii, T. Matsuura and T. Yomo, ACS Chem. Biol., 2015, 10, 1694-1701.

34 S. Benke, D. Roderer, B. Wunderlich, D. Nettels, R. Glockshuber and B. Schuler, Nat. Commun., 2015, 6, 6198. 